\title{
Analytical solutions for transient and steady state beam loading in arbitrary traveling wave accelerating structures
}

\author{
A. Lunin and V. Yakovlev \\ Fermilab, P.O. Box 500, Batavia, Illinois 60510, USA \\ A. Grudiev \\ CERN, CH-1211 Geneva-23, Switzerland \\ (Received 15 December 2010; published 19 May 2011)
}

\begin{abstract}
Analytical solutions are derived for both transient and steady state gradient distributions in the traveling wave (TW) accelerating structures with arbitrary variation of parameters over the structure length. The results of the unloaded and beam loaded cases are presented. Finally, the exact analytical shape of the rf pulse waveform was found in order to apply the transient beam loading compensation scheme during the structure filling time. The obtained theoretical formulas were cross-checked by direct numerical simulations on the CLIC main linac accelerating structure and demonstrated a good agreement. The proposed methods provide a fast and reliable tool for the initial stage of the TW structure analysis.
\end{abstract}

DOI: 10.1103/PhysRevSTAB.14.052001

PACS numbers: 29.20.Ej

\section{INTRODUCTION}

The steady state theory of beam loading in electron linear accelerators was developed in the 1950 s by a number of authors both for constant impedance [1-3] and constant gradient [4] accelerating structures. They considered the equation for energy conservation in a volume between any two cross sections; the power gained by the beam or lost in the walls due to the Joule effect results in a reduction of the power flow. Later on, transient behavior was studied following a similar approach, but in this case, in addition to the power dissipated in the walls and gained by the beam, the transient change in the energy stored in the volume contributes to the power flow variation along the structure. Again, only constant impedance [5-7] or constant gradient $[8,9]$ accelerating structures were considered.

However, traveling wave accelerating structures with arbitrary (neither constant impedance nor constant gradient) geometrical variations over the length are widely used today in order to optimize the acceleration structure and linac performance $[10,11]$. The relationships between structure length, input, and average accelerating gradients are obtained by solving the energy conservation equation numerically. For the first time an analytical solution of the gradient profile in a loaded arbitrary traveling wave (TW) structure was recently proposed in [12] but for the steady state regime only. The comprehensive numerical analysis of an arbitrary TW structure including the effects of a

Published by the American Physical Society under the terms of the Creative Commons Attribution 3.0 License. Further distribution of this work must maintain attribution to the author(s) and the published article's title, journal citation, and DOI. signal dispersion was recently published in [13] using the circuit model and mode matching technique.

In this paper, generalized analytical solutions of the gradient distribution in the TW accelerating structure with an arbitrary variation of parameters over the structure length are presented for both steady state and transient regimes. It is based on the method suggested earlier by one of the coauthors [14] and is similar to the classical approach [1-9]. Finally, a simple analytical relation is derived that allows the input power ramp needed to create, at the end of the filling time, the field distribution inside the TW structure that coincides to the loaded field distribution in the presence of the beam to be determined. The compact analytical formulas so obtained give us a better understanding of the physics of TW structures and provide a tool for a fast preliminary structure optimization.

The following definitions are used throughout the paper: (i) $P$-power flow through the structure cross section; (ii) $W^{*}$-stored energy per unit length; (iii) $\omega$-circular frequency; (iv) $Q^{*}$ - quality factor; (v) $G^{*}$-loaded accelerating gradient; (vi) $\tilde{G}^{*}$ - unloaded accelerating gradient; (vii) $I$-beam current; (viii) $v_{g}{ }^{*}$-group velocity; (ix) $\rho^{*}$-normalized shunt impedance, often called $R / Q$, where $R$ is the shunt impedance per unit length; and (x) $z$-longitudinal coordinate. The symbol $*$ denotes that continuous parameters are averaged over the structure period and represent the effective values of an individual cell.

The following assumptions are used: (a) the structure is perfectly matched at both ends and has no internal reflections; (b) all dispersion effects that limit field rise time: $t_{r} \gg c / \omega v_{g}$, where $c$ is the speed of light, are neglected; (c) time separation between two neighboring bunches and 
TABLE I. Parameters of the CLIC main linac accelerating structure.

\begin{tabular}{lc}
\hline \hline Average loaded accelerating gradient & $100 \mathrm{MV} / \mathrm{m}$ \\
Frequency & $12 \mathrm{GHz}$ \\
rf phase advance per cell & $2 \pi / 3 \mathrm{rad}$ \\
First, middle, and last cell group velocity & $1.2,0.83 \%$ of c \\
First, middle, and last cell $Q$ factor $(\mathrm{Cu})$ & $5536,5635,5738$ \\
First, middle, and last cell normalized shunt impedance & $14587,16220,17954 \Omega / \mathrm{m}$ \\
Number of regular cells & 26 \\
Structure length including couplers & $230 \mathrm{~mm}$ \\
Bunch spacing & $0.5 \mathrm{~ns}$ \\
Bunch population & $3.7 \times 10^{9}$ \\
Number of bunches in the train & 312 \\
Rise time & $22 \mathrm{~ns}$ \\
Filling time & $67 \mathrm{~ns}$ \\
Peak input power & $61.3 \mathrm{MW}$ \\
\hline \hline
\end{tabular}

time of flight of the beam through the structure are much less than the filling time of the structure.

\section{STEADY STATE REGIME}

The basic traveling wave structure relations are

$$
\begin{gathered}
P=W v_{g} \\
W=\frac{G^{2}}{\omega \rho} .
\end{gathered}
$$

Energy conservation including wall losses and the interaction with the beam gives

$$
\frac{d P}{d z}=-\frac{W \omega}{Q}-G I
$$

Using Eq. (2.2) in the derivation of the power flow Eq. (2.1) yields

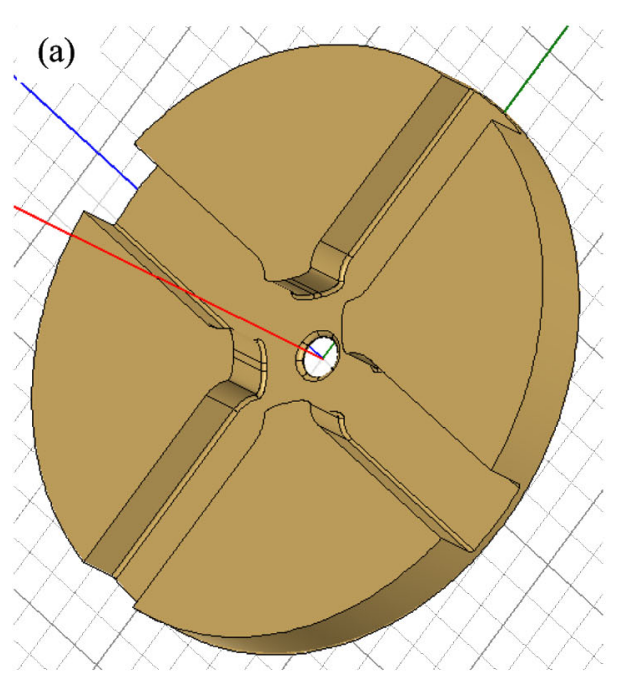

$$
\begin{aligned}
\frac{d P}{d z} & =W \frac{d v_{g}}{d z}+v_{g} \frac{d W}{d z} \\
& =\frac{G^{2}}{\omega \rho} \frac{d v_{g}}{d z}+\frac{v_{g}}{\omega}\left[\frac{2 G}{\rho} \frac{d G}{d z}-\frac{G^{2}}{\rho^{2}} \frac{d \rho}{d z}\right]
\end{aligned}
$$

Substituting Eq. (2.4) into Eq. (2.3) and using Eq. (2.2) results in the first order nonhomogeneous differential equation with variable coefficients:

$$
\frac{d G}{d z}=-G(z) \alpha(z)-\beta(z),
$$

where $\alpha(z)=\frac{1}{2}\left[\frac{1}{v_{g}} \frac{d v_{g}}{d z}-\frac{1}{\rho} \frac{d \rho}{d z}+\frac{\omega}{v_{g} Q}\right], \beta(z)=I \frac{\omega \rho}{2 v_{g}}$. The solution of the nonhomogeneous differential Eq. (2.5), $G(z)$, can be presented as a product of the solution of the homogeneous equation $\tilde{G}(z)$ and a function $C(z)$ :

$$
G(z)=\tilde{G}(z) \cdot C(z)
$$

(b)

(c)

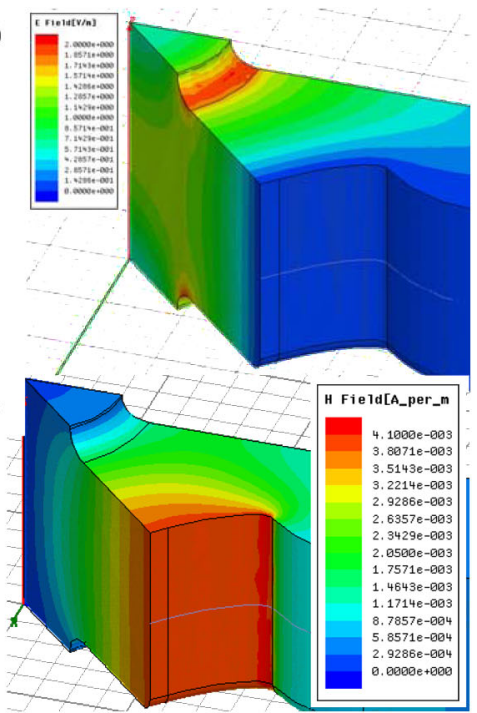

FIG. 1. Individual cell geometry of the CLIC main linac accelerating structure with strong waveguide high order modes damping (a), HFSS simulations of the surface electric (b), and magnetic (c) fields are shown. 


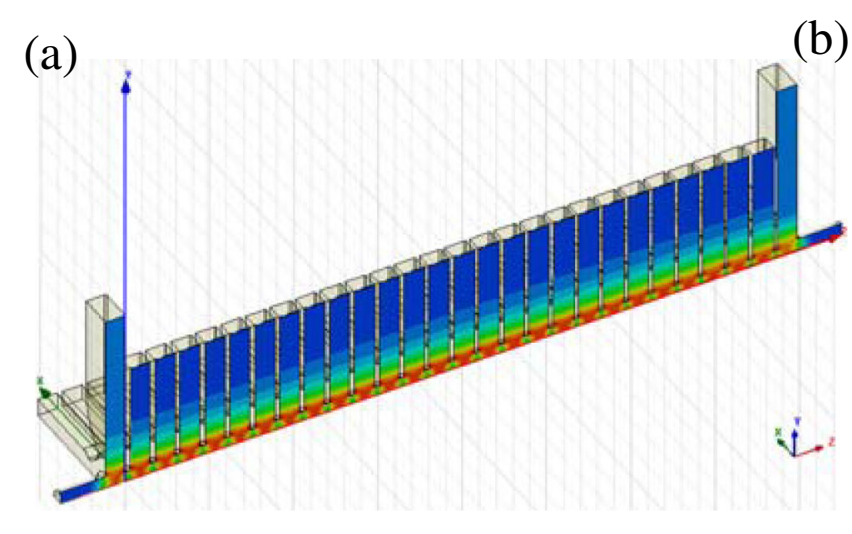

(b)

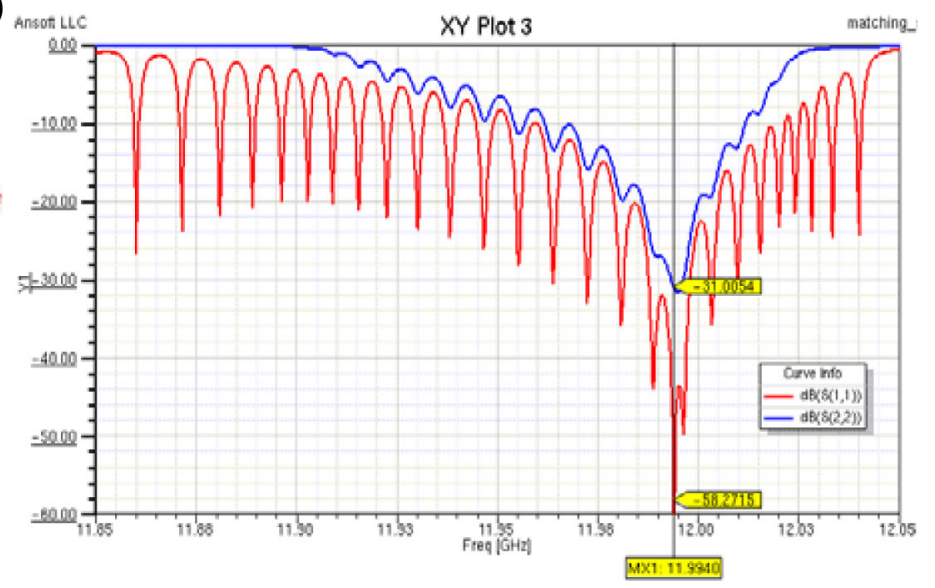

(c) $\mathrm{f}=11.99 \mathrm{GHz}$
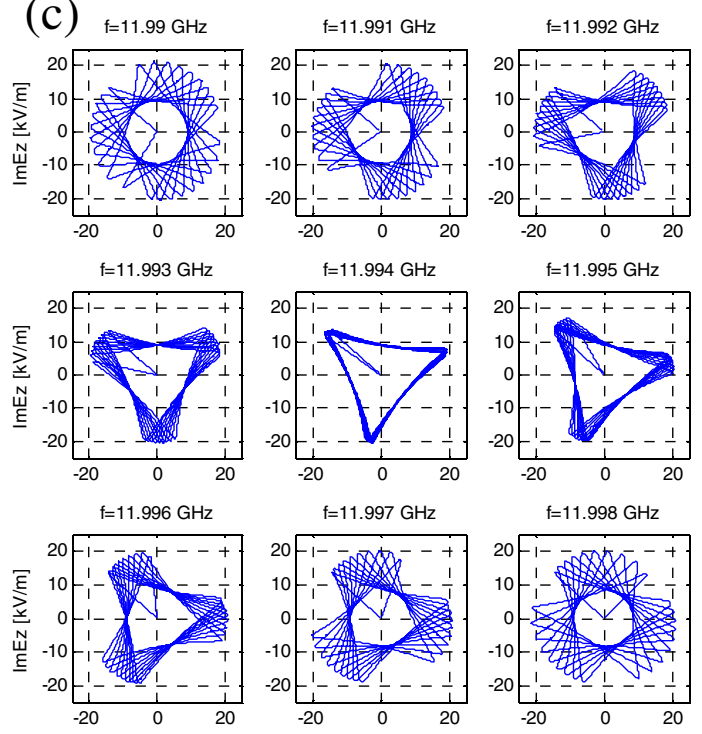

(d)

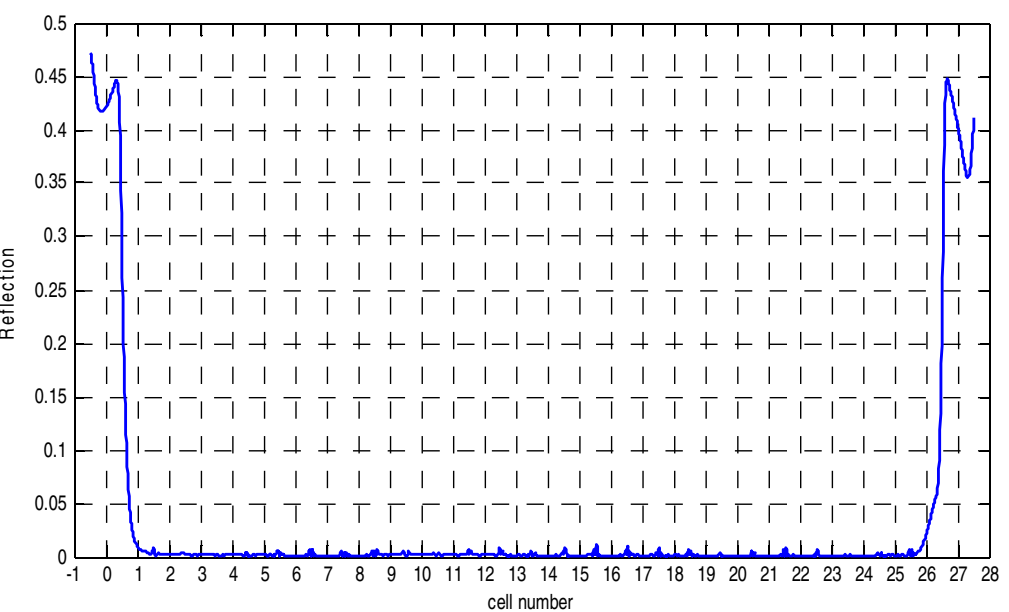

FIG. 2. The HFSS simulation of the full CLIC accelerating structure. Electric field profile (a), input $\left(S_{11}\right.$, red curve) and output $\left(S_{22}\right.$, blue curve) couplers matching (b), phase advance per cell versus frequency (c), and internal reflections (standing wave ratio) in the structure (d) are shown. The phase advance per cell is equal to $120^{\circ}$ and both couplers are matched better than $-30 \mathrm{~dB}$ level at operating frequency of $11.994 \mathrm{GHz}$.

where

$$
\frac{d \tilde{G}}{d z}=-\tilde{G}(z) \alpha(z)
$$

Substituting Eq. (2.6) into Eq. (2.5) and using Eq. (2.7) yields

$$
\frac{d C(z)}{d z}=-\frac{\beta(z)}{\tilde{G}(z)}
$$

Integrating Eq. (2.8) gives

$$
C(z)=-\int_{0}^{z} \frac{\beta\left(z^{\prime}\right)}{\tilde{G}\left(z^{\prime}\right)} d z^{\prime}+C_{1},
$$

where the constant $C_{1}=1$ [taking into account the initial condition $G(0)=\tilde{G}(0)]$ and $z^{\prime}$ is a local integration variable.
Therefore the general solution of Eq. (2.5) is

$$
G(z)=\tilde{G}(z)\left[-\int_{0}^{z} \frac{\beta\left(z^{\prime}\right)}{\tilde{G}\left(z^{\prime}\right)} d z^{\prime}+1\right] .
$$

The solution for the homogeneous Eq. (2.7) is

$$
\tilde{G}(z)=G_{0} e^{-\int_{0}^{z} \alpha\left(z^{\prime}\right) d z^{\prime}},
$$

where $G_{0}=G(0)$ is a gradient at the beginning of accelerating structure and can be found from initial conditions:

$$
G_{0}=\sqrt{\frac{\omega \rho(0) P_{0}}{v_{g}(0)}}
$$

where $P_{0}$ is input rf power.

The integral of function $\alpha(z)$ can be simplified using analytical solutions: 


$$
\begin{aligned}
\int_{0}^{z} \alpha\left(z^{\prime}\right) d z^{\prime}= & \frac{1}{2}\left[\ln \left(\frac{v_{g}(z)}{v_{g}(0)}\right)-\ln \left(\frac{\rho(z)}{\rho(0)}\right)\right. \\
& \left.+\int_{0}^{z} \frac{\omega}{v_{g}\left(z^{\prime}\right) Q\left(z^{\prime}\right)} d z^{\prime}\right]
\end{aligned}
$$

Finally we can rewrite Eq. (2.10) as

$$
\begin{aligned}
\tilde{G}(z) & =G_{0} \sqrt{\frac{v_{g}(0)}{v_{g}(z)}} \sqrt{\frac{\rho(z)}{\rho(0)}} e^{-(1 / 2) \int_{0}^{z}\left(\omega / v_{g}\left(z^{\prime}\right) Q\left(z^{\prime}\right)\right) d z^{\prime}} \\
& =G_{0} g(z) .
\end{aligned}
$$

Equations (2.13) and (2.9) give us an expression for the loaded gradient:

$$
\begin{aligned}
G(z) & =\tilde{G}(z)\left[1-\int_{0}^{z} \frac{I}{\tilde{G}\left(z^{\prime}\right)} \frac{\omega \rho\left(z^{\prime}\right)}{2 v_{g}\left(z^{\prime}\right)} d z^{\prime}\right] \\
& =G_{0} g(z)-g(z) \int_{0}^{z} \frac{I}{g\left(z^{\prime}\right)} \frac{\omega \rho\left(z^{\prime}\right)}{2 v_{g}\left(z^{\prime}\right)} d z^{\prime} .
\end{aligned}
$$

The first term on the right-hand side of Eq. (2.14) is the solution of the homogeneous equation for the unloaded gradient obtained above in Eq. (2.13). The second term is the so-called beam induced gradient which is the difference between the loaded and unloaded gradient distributions.

Parameters of the CLIC main linac accelerating structure are summarized in Table I [11]. They have been used to compare an accurate solution for an arbitrary variation of the TW structure parameters given by Eq. (2.14) to an approximate solution given in [4], where it has been assumed that the shunt impedance and $Q$ factor are constant in the range over which the group velocity changes and that they are both equal to their respective averages over the structure.

The unloaded gradient has been calculated for a 3D model of the structure using ANSOFT HFSS [15], a frequency-domain finite-element code which takes into account internal reflections [11]. First of all, the parameters of individual cells were calculated for the given phase advance, shunt impedance, group velocity, and maximum EM-field strength on the surface. The result of individual cell optimization is shown in Fig. 1. Next, the input and output rf couplers were designed in order to match the TW structure with feeding waveguide and rf loads. The detailed procedure of rf coupler design using ANSOFT HFSS code is described in [16]. After that, we made the simulation of full CLIC main linac accelerating structure and verified rf phase advance per cell and internal reflections using the well-known "Kroll's" method [17] (see Fig. 2). Finally, we derived the secondary values (stored energy and rf power flow per cell) necessary for the unloaded gradient calculation.

Both the loaded and unloaded gradients are shown in Fig. 3 for an input rf power of $61.3 \mathrm{MW}$ which corresponds to an average loaded gradient of $100 \mathrm{MV} / \mathrm{m}$. There is

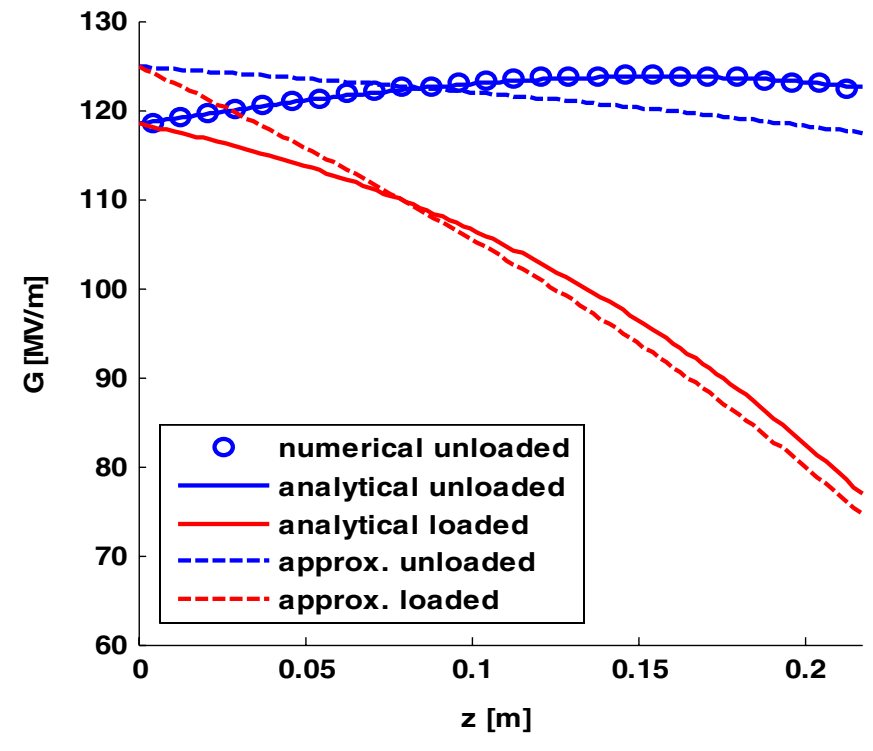

FIG. 3. Loaded (red) and unloaded (blue) gradients calculated accurately (solid) and approximately (dashed) for the CLIC main linac accelerating structure. In blue circles, the unloaded gradient calculated numerically is shown.

clearly a very good agreement between the accurate analytical solution and the numerical simulation. In contrast, the approximate solution is quite different from the accurate solution due mainly to a significant $(\sim 30 \%)$ variation of the shunt impedance along the structure, see Table I.

\section{TRANSIENT REGIME}

The transient regime can also be derived analytically. The instantaneous energy conservation is given by

$$
\frac{\partial W}{\partial t}=-\frac{d P}{d z}-\frac{W \omega}{Q}-G I .
$$

Substituting Eqs. (2.2) and (2.4) into Eq. (3.1) yields

$$
\frac{\partial G}{\partial t}=-\frac{G}{2} \frac{d v_{g}}{d z}-v_{g} \frac{d G}{d z}+\frac{v_{g} G}{2 \rho} \frac{d \rho}{d z}-\frac{\omega G}{2 Q}-\frac{\omega \rho}{2} I .
$$

We assume the following initial conditions:

$$
G(0, t)=G_{0}(t), \text { at } z=0 \quad G(z, 0)=0 \text {, at } t=0 .
$$

Using the Laplace transformation of a function $G(t)$,

$$
\begin{aligned}
& \hat{G}(p)=L\{G(t)\}=\int_{0}^{\infty} e^{-p t} G(t) d t, \\
& t \geq 0, \text { its differentiation property: } L\left\{\frac{d G}{d t}\right\}=p \hat{G}-G(z, 0),
\end{aligned}
$$

and taking into account Eq. (3.3) we can write Eq. (3.2) as follows:

$$
p \hat{G}=-\frac{\hat{G}}{2} \frac{d v_{g}}{d z}-v_{g} \frac{d \hat{G}}{d z}+\frac{v_{g} \hat{G}}{2 \rho} \frac{d \rho}{d z}-\frac{\omega \hat{G}}{2 Q}-\frac{\omega \rho}{2} \hat{I} .
$$


First, we consider the unloaded case $(I=0)$. In this case Eq. (3.4) becomes a homogeneous differential equation:

$$
\frac{d \hat{\tilde{G}}}{d z}=-\hat{\tilde{G}}(z, p) \hat{\alpha}(z, p),
$$

where $\hat{\alpha}(z, p)=\frac{1}{2}\left[\frac{1}{v_{g}} \frac{d v_{g}}{d z}-\frac{1}{\rho} \frac{d \rho}{d z}+\frac{\omega}{v_{g} Q}+\frac{2 p}{v_{g}}\right]$. The solution of Eq. (3.5), obtained in a similar manner to the solution of Eq. (2.7), is

$$
\begin{aligned}
\hat{\tilde{G}}(z, p) & =\hat{\tilde{G}}(0, p) e^{-\int_{0}^{z} \hat{\alpha}\left(z^{\prime}, p\right) d z^{\prime}} \\
& =\hat{\tilde{G}}(0, p) g(z) e^{-p \int_{0}^{z}\left[d z^{\prime} / v_{g}\left(z^{\prime}\right)\right]},
\end{aligned}
$$

where $g(z)$ is defined in Eq. (2.13). The time-domain solution of Eq. (3.6) is obtained by applying the inverse Laplace transformation and its time shifting property: $L^{-1}\left\{F(p) e^{-p \tau}\right\}=f(t-\tau) H(t-\tau)$, where $H(t-\tau)$ is the Heaviside step function and

$$
\tau(z)=\int_{0}^{z} \frac{d z^{\prime}}{v_{g}\left(z^{\prime}\right)}
$$

is the signal time delay. Thus, the distribution of the unloaded gradient in time domain along the structure is

$$
\tilde{G}(z, t)=G_{0}[t-\tau(z)] g(z) H[t-\tau(z)],
$$

or taking into account Eqs. (2.11) and (2.13) it can be expressed as a function of the input $\mathrm{rf}$ power:

$$
\begin{aligned}
\tilde{G}(z, t)= & \sqrt{P_{0}[t-\tau(z)]} \sqrt{\frac{\omega \rho(z)}{v_{g}(z)}} e^{-(1 / 2) \int_{0}^{z}\left[\omega / v_{g}\left(z^{\prime}\right) Q\left(z^{\prime}\right)\right] d z^{\prime}} \\
& \times H[t-\tau(z)] .
\end{aligned}
$$

The solution of nonhomogeneous Eq. (3.4) is obtained in a similar manner to the solution of Eq. (2.5) as a product of the solution to the homogeneous equation $\hat{\tilde{G}}(z, p)$ and a function $\hat{C}(z, p)$ :

$$
\hat{G}(z, p)=\hat{\tilde{G}}(z, p) \cdot \hat{C}(z, p) .
$$

Then Eq. (3.4) becomes

$$
\frac{d \hat{G}}{d z}=\frac{d \hat{\tilde{G}}}{d z} \hat{C}+\frac{d \hat{C}}{d z} \hat{\tilde{G}}=-\hat{G}(z, p) \hat{\alpha}(z, p)-\hat{\beta}(z, p),
$$

where $\hat{\beta}(z, p)=\hat{I} \frac{\omega \rho}{2 v_{g}}$.

Substituting Eqs. (3.5) and (3.10) into Eq. (3.11) yields

$$
\hat{\tilde{G}} \frac{d \hat{C}}{d z}=-\hat{\beta}(z, p)
$$

and furthermore using Eqs. (3.6) and (3.7),

$$
\hat{\tilde{G}}(0, p) g(z) e^{-p \tau(z)} \frac{d \hat{C}}{d z}=-\hat{\beta}(z, p) .
$$

The solution of Eq. (3.13) can be obtained by integration in the form

$$
\hat{\tilde{G}}(0, p) \hat{C}(z, p)=-\int_{0}^{z} \frac{\hat{\beta}\left(z^{\prime}, p\right)}{g\left(z^{\prime}\right)} e^{p \tau\left(z^{\prime}\right)} d z^{\prime}+\hat{C}_{1}(p),
$$

where $\hat{C}_{1}(p)=\hat{\tilde{G}}(0, p)$ [taking into account the initial condition $\hat{G}(0, p)=\hat{\tilde{G}}(0, p)]$. Note, that $g(z)>0$. Finally, the general solution of Eq. (3.4) is derived using Eqs. (3.10) and (3.14):

$$
\begin{aligned}
\hat{G}(z, p) & =\hat{\tilde{G}}(0, p) \hat{C}(z, p) g(z) e^{-p \tau(z)} \\
& =\left[\hat{\tilde{G}}(0, p)-\int_{0}^{z} \frac{\hat{\beta}\left(z^{\prime}, p\right)}{g\left(z^{\prime}\right)} e^{p \tau\left(z^{\prime}\right)} d z^{\prime}\right] g(z) e^{-p \tau(z)} \\
& =\hat{\tilde{G}}(z, p)-g(z) \int_{0}^{z} \frac{\hat{\beta}\left(z^{\prime}, p\right)}{g\left(z^{\prime}\right)} e^{-p\left[\tau(z)-\tau\left(z^{\prime}\right)\right]} d z^{\prime} .
\end{aligned}
$$
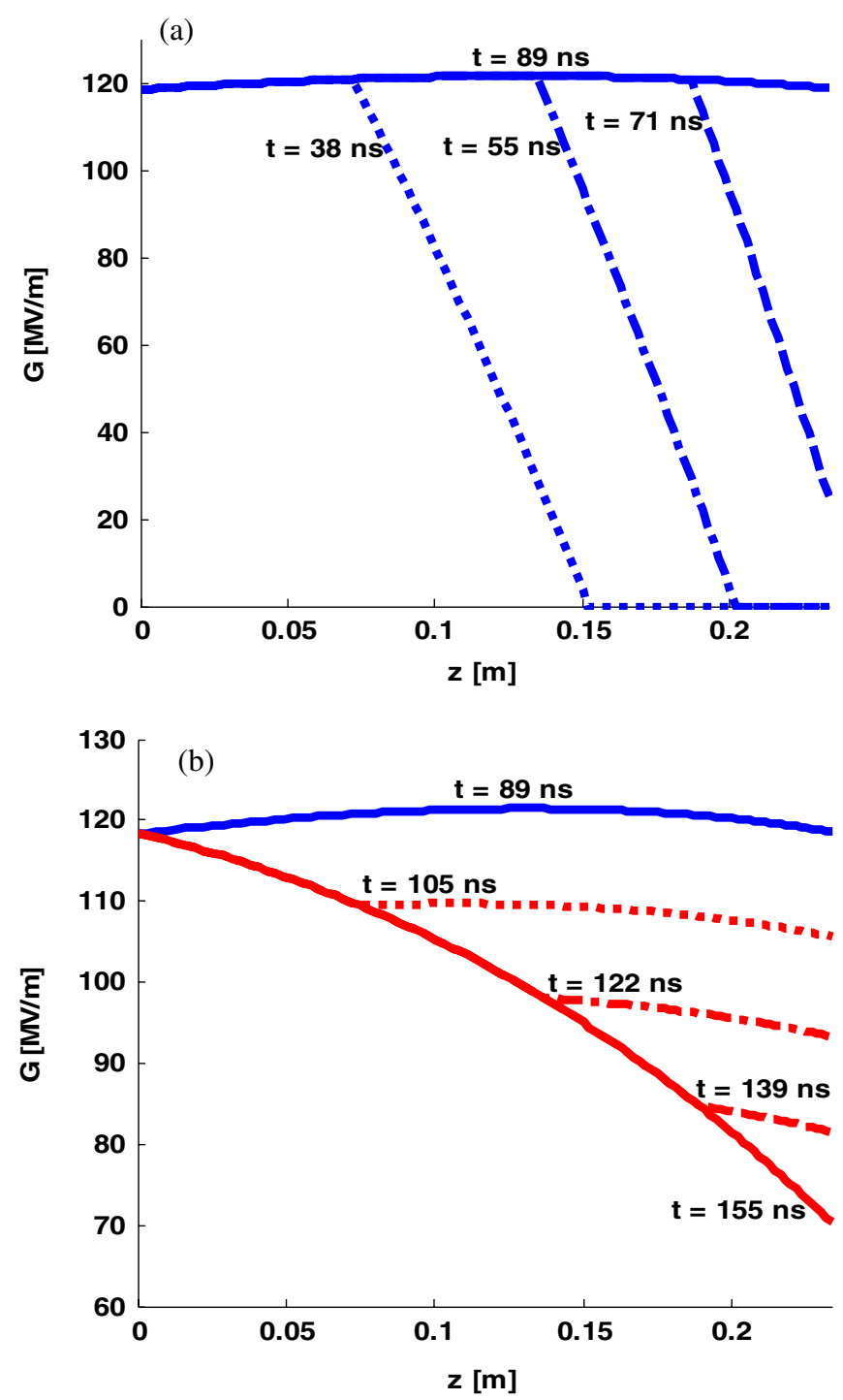

FIG. 4. The instantaneous unloaded (blue) and loaded (red) gradient distributions along the structure at different moments of time during the transient related to structure filling (a) and to the beam injection (b). The steady state solutions are shown as well (solid lines). 
Thus the time-dependent solution of Eq. (3.1) is obtained by applying the inverse Laplace transform to Eq. (3.15). Here again the time shifting property has been used:

$$
\begin{aligned}
G(z, t)= & G_{0}[t-\tau(z)] g(z) H[t-\tau(z)] \\
& -g(z) \int_{0}^{z} \frac{I\left[t-\tau(z)+\tau\left(z^{\prime}\right)\right] H\left[t-\tau(z)+\tau\left(z^{\prime}\right)\right]}{g\left(z^{\prime}\right)} \\
& \times \frac{\omega \rho\left(z^{\prime}\right)}{2 v_{g}\left(z^{\prime}\right)} d z^{\prime},
\end{aligned}
$$

where $\tau(z)$ is a function of the coordinate $z$ and given by Eq. (3.7).

The first term on the right-hand side of Eq. (3.16) is the solution of the homogeneous equation for the unloaded gradient obtained above in Eq. (3.8) or Eq. (3.9) in terms of the input power. The second term is the so-called beam induced gradient which is the difference between the loaded and unloaded gradient distributions.

For the CLIC main linac accelerating structure with the parameters from Table I, the time-dependent solution given by Eq. (3.16) during the transient related to structure filling and to beam injection is illustrated in Figs. 4(a) and 4(b), respectively. In Fig. 5, the corresponding input power and beam-current time dependences are shown together with the unloaded, loaded, and beam voltages defined as

$$
\begin{gathered}
V(t)=\int_{0}^{L} G(z, t) d z, \quad \tilde{V}(t)=\int_{0}^{L} \tilde{G}(z, t) d z, \\
V_{b}(t)=V(t)-\tilde{V}(t),
\end{gathered}
$$

respectively, where $L$ is the structure length. In order to mitigate unwanted dispersion effects the signal front width is limited by a slow amplitude rise, with the rise time $t_{r}=22 \mathrm{~ns} \gg c / \omega v_{g}$. The sum of the signal rise time $t_{r}$

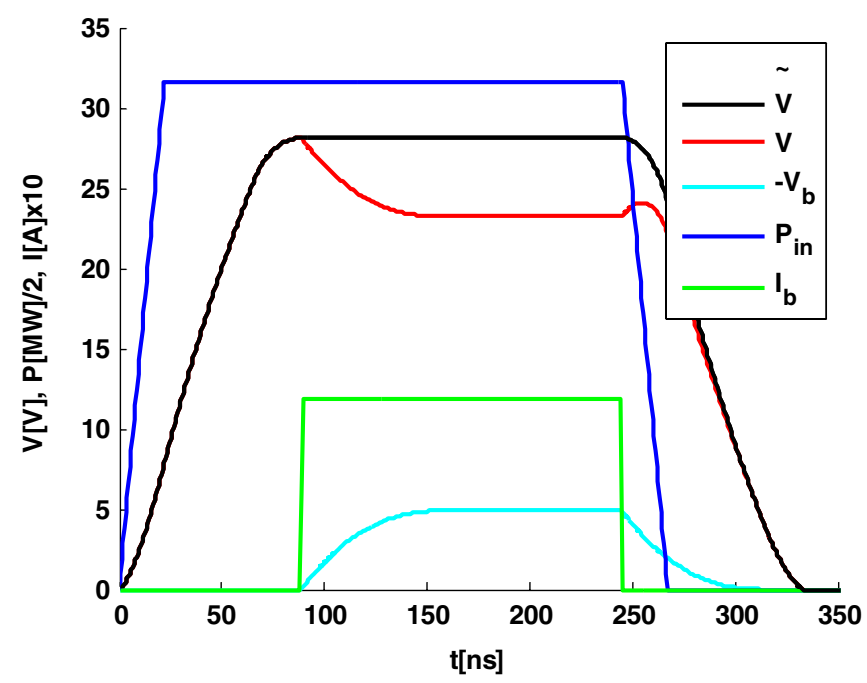

FIG. 5. The time dependence of the input $\mathrm{rf}$ power (blue) with a rise time of $22 \mathrm{~ns}$, beam current (green), and the corresponding unloaded (black), loaded (red), and beam (light blue) voltages are shown. and the structure filling time $t_{f}=\tau(L)=66.7 \mathrm{~ns}$ form the overall time of $89 \mathrm{~ns}$ corresponding to a transient of a cavity excitation. The total beam pulse length $t_{b}$ is $312 \times 0.5=156 \mathrm{~ns}$ and the beam current $I=e N_{e} f_{b}=$ $1.6 \times 10^{-19} \cdot 3.72 \times 10^{9} /\left(0.5 \times 10^{-9}\right)$ is $1.2 \mathrm{~A}$ [11].

A transient change in the loaded voltage just after the beam injection causes energy spread along a multibunch beam train. One possible method of transient beam loading compensation in TW structures is presented in the next section.

\section{COMPENSATION OF THE TRANSIENT BEAM LOADING}

The idea of transient beam loading compensation was proposed in 1993 at SLAC (USA) [18], where a linear ramp of the input rf amplitude has been applied to compensate the bunch-to-bunch energy variation to first order. Later a sophisticated numerical algorithm for beam loading compensation was developed in the framework of the Next Linear Collider project in order to calculate the precise profile of the rf pulse wavefront [19]. Recently, the effectiveness of this method of transient beam loading compensation has been experimentally verified at KEK (Japan) [20,21].

In this paper, the exact modification of the input power during a filling time $t_{f}$ needed to set the gradient distribution at the beam injection time equal to the steady state loaded gradient solution $G(z)$ is calculated. Thus, the loaded voltage remains flat during the time when the beam is on because the transient related to the beam injection is fully compensated by the transient of the cavity

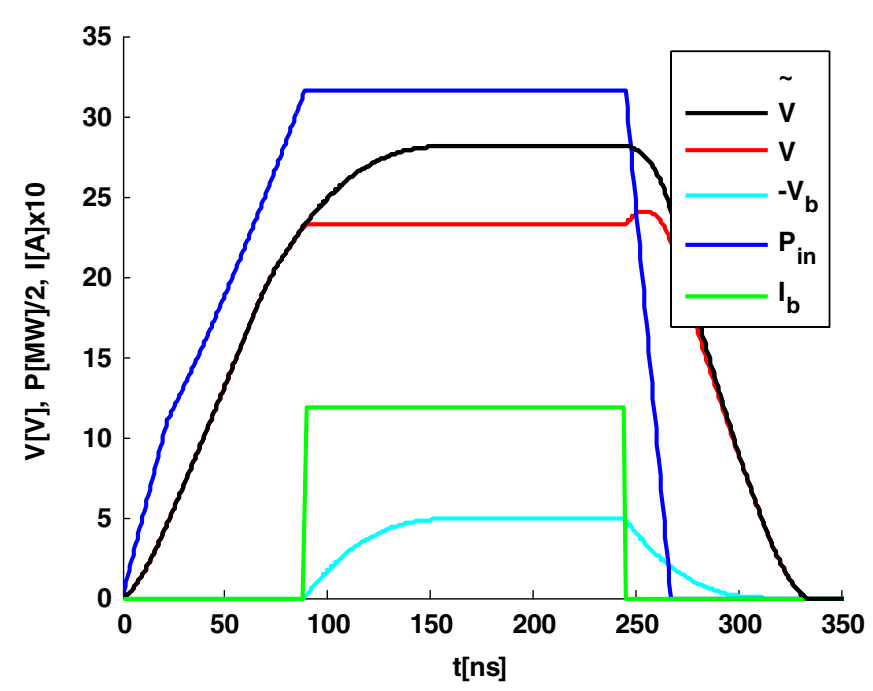

FIG. 6. The input rf pulse profile with $22 \mathrm{~ns}$ rise time and ramp-up during the filling time for the transient beam loading compensation is shown in blue. Beam current injected exactly at the end of the ramp is shown in green. The corresponding unloaded, loaded, and beam voltages are shown in black, red, and light blue, respectively. 
excitation itself (at least in the framework of the applied analytical model).

Based on Eq. (3.8), the instantaneous gradient distribution at the moment of injection $t=t_{f}$ is

$$
G\left(z, t_{f}\right)=\tilde{G}\left(z, t_{f}\right)=G_{0}\left[t_{f}-\tau(z)\right] g(z) .
$$

At the same time, the steady state beam loaded solution is expressed by (2.14). Equating Eq. (4.1) and Eq. (2.14), the required time dependence for the input gradient $G_{0}(t)$ during the filling time is obtained:
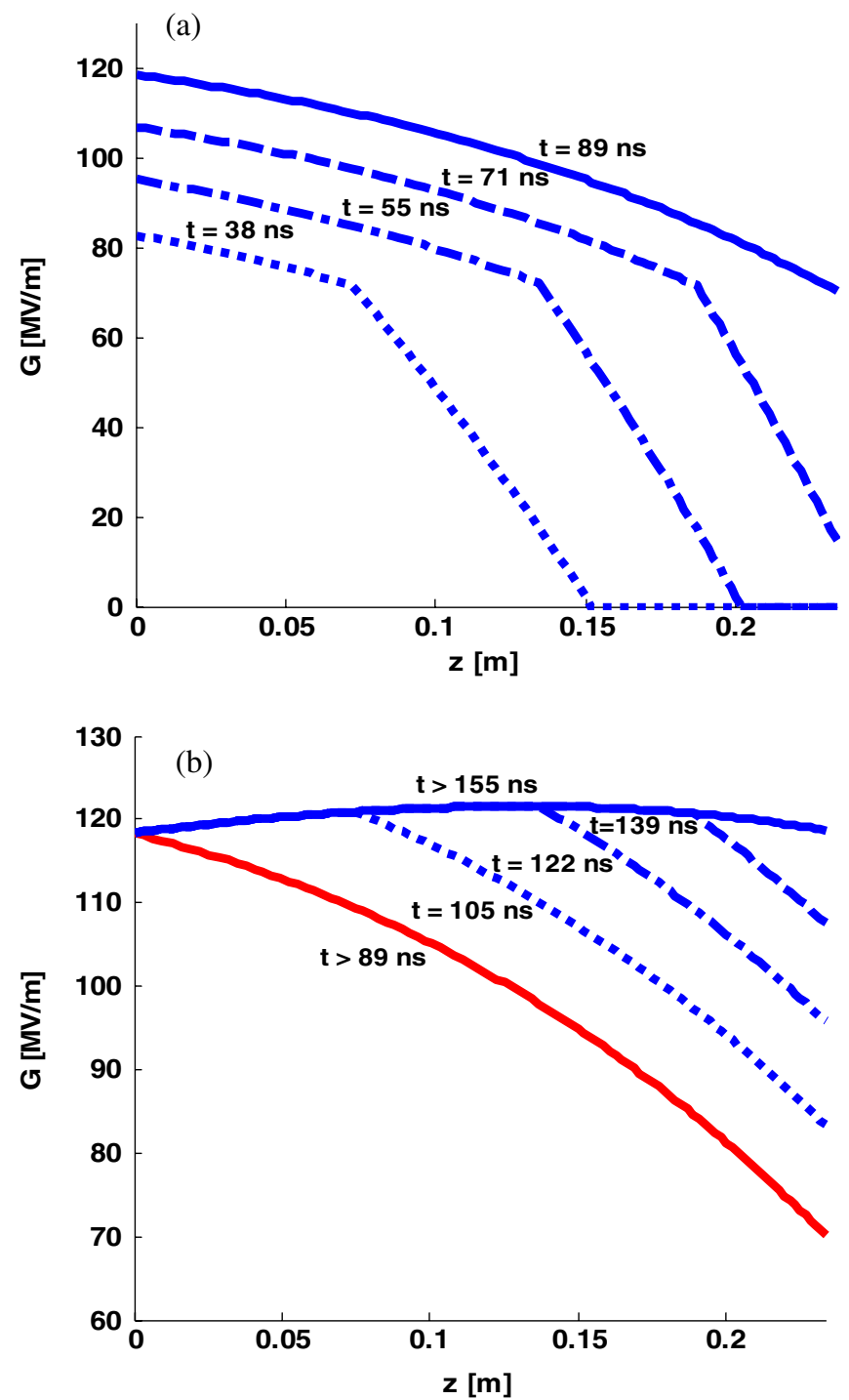

FIG. 7. The instantaneous unloaded gradient distribution along the structure at different moments of time is presented in (a). Special correction to the input rf pulse was applied (see Fig. 6). In (b), the instantaneous unloaded gradient at different moments of time after beam injection is shown in blue. Solid lines represent the steady state distributions for loaded (red) and unloaded (blue) gradients. The beam injection time is $89 \mathrm{~ns}$.
$G_{0}\left[t_{f}-\tau(z)\right] g(z)=G_{0}\left(t_{f}\right) g(z)-g(z) \int_{0}^{z} \frac{I}{g\left(z^{\prime}\right)} \frac{\omega \rho\left(z^{\prime}\right)}{2 v_{g}\left(z^{\prime}\right)} d z^{\prime}$,

where $G_{0}\left(t_{f}\right)$ is the steady state value of the input gradient after injection. The input gradient in Eq. (4.2) indirectly depends on time. Introducing the function $z(t)$ as a solution of the following integral equation,

$$
t(z)=\int_{z}^{L} \frac{d z^{\prime}}{v_{g}\left(z^{\prime}\right)}
$$

Eq. (4.2) becomes an explicit function of time:

$$
G_{0}(t)=G_{0}\left(t_{f}\right)-\int_{0}^{z(t)} \frac{I}{g\left(z^{\prime}\right)} \frac{\omega \rho\left(z^{\prime}\right)}{2 v_{g}\left(z^{\prime}\right)} d z^{\prime}
$$

An expression for the input $\mathrm{rf}$ power is derived using Eq. (2.11):

$$
\begin{aligned}
P_{0}(t)= & P_{0}\left(t_{f}\right)\left[1-\sqrt{\frac{v_{g}(0)}{\omega \rho(0) P_{0}\left(t_{f}\right)}}\right. \\
& \left.\times \int_{0}^{z(t)} \frac{I}{g\left(z^{\prime}\right)} \frac{\omega \rho\left(z^{\prime}\right)}{2 v_{g}\left(z^{\prime}\right)} d z^{\prime}\right]^{2}
\end{aligned}
$$

where $P_{0}\left(t_{f}\right)$ is the steady state value of the input rf power after injection.

The solution of Eq. (4.5) is shown in Fig. 6 (blue) together with the beam current (green) injected exactly at the end of the ramp and the corresponding unloaded (black), loaded (red), and beam (light blue) voltages. The gradient distribution at different moments of time is presented for the compensated case in Figs. 7(a) and 7(b) for the structure filling transient and the beam injection transient, respectively.

\section{SUMMARY}

Analytical expressions for unloaded and loaded gradient distributions in traveling wave structures with arbitrary variation of parameters were derived in steady state and in transient. They were applied to the case of the CLIC main linac accelerating structure. The analytical solution agrees very well with the numerical solution obtained using finite-element code. On the other hand, it differs from the approximate solution obtained using expressions derived earlier in [4]. Finally, the exact analytical solution was found for the wavefront of input rf pulse which theoretically provides exact compensation of the beam loading effect. The derived analytical formulas are very useful during the preliminary stages of structure design and later for structure efficiency optimization. 


\section{ACKNOWLEDGMENTS}

The authors are thankful to W. Wuensch and N. Shipman for carefully reading the manuscript.

[1] K. Johnsen, Proc. Phys. Soc. London Sect. B 64, 1062 (1951).

[2] G. Saxon, Proc. Phys. Soc. London Sect. B 67, 705 (1954).

[3] R. B. Neal, J. Appl. Phys. 29, 1019 (1958); W. W. Hansen Laboratories, M. L. Report No. 379, Stanford, 1957.

[4] R. B. Neal, W. W. Hansen Laboratories, M. L. Report No. 513, Stanford, 1958.

[5] R. B. Neal, M. L. Report No. 388, 1957.

[6] J. E. Leiss, NBS Internal Report 1958.

[7] J.E. Leiss, Beam Loading and Transient Behavior in Travelling Wave Electron Linear Accelerators, in Linear Accelerators, edited by A. Septier and P. M. Lapostolle (North-Holland Publishing Company, Amsterdam, 1969).

[8] L. Burnod, LAL Report No. 17, Orsay, 1961.

[9] J. W. Wang, Ph.D. dissertation, Stanford University, 1989 [Report No. SLAC-339, 1989].

[10] R.H. Miller et al., in Proceedings of the XVIII International Linac Conference (LINAC96) (CERN, Geneva, Switzerland, 1996).
[11] A. Grudiev and W. Wuensch, in Proceedings of the XXV Linear Accelerator Conference (LINAC10) (JACoW, 2010), MOP068, http://www.jacow.org.

[12] G. Guignard and J. Hagel, Phys. Rev. ST Accel. Beams 3, 042001 (2000).

[13] R. M. Jones, V. A. Dolgashev, and J. W. Wang, Phys. Rev. ST Accel. Beams 12, 051001 (2009).

[14] M. M. Karliner, O. A. Nezhevenko, B. M. Fomel, and V. P. Yakovlev, Report No. INP 86-146, 1986 (in Russian).

[15] ANSOFT HFSS, www.ansoft.com.

[16] A. Lounine and T. Higo, in Proceedings of the 14th Symposium on Accelerator Science and Technology (National Laboratory for High Energy Physics (KEK), Tsukuba, Japan, 2003).

[17] N. M. Kroll et al., in Proceedings of the 20th International Linac Conference, Monterey, CA, 2000 (SLAC, Menlo Park, CA, 2000).

[18] K. A. Thompson and R. D. Ruth, in Proceedings of the Particle Accelerator Conference, Washington, DC, 1993 (IEEE, New York, 1993).

[19] C. Nantista and C. Adolphsen, SLAC NLC-Note Report No. 25, 1997.

[20] M. Satoh et al., Nucl. Instrum. Methods Phys. Res., Sect. A 538, 116 (2005).

[21] M. Satoh et al., Phys. Rev. ST Accel. Beams 12, 013501 (2009). 